\title{
Paramètres de reproduction et de dynamique de population de la chèvre locale de Mbanza-Ngungu en République démocratique du Congo
}

\author{
Richard Gasigwa Sabimana ${ }^{1 *}$ Patrick Baenyi Simon ${ }^{2}$ \\ Roger Kizungu Vumilia ${ }^{3}$
}

\section{Mots-clés}

Caprin, petit ruminant, reproduction, dynamique des populations, République démocratique du Congo

Submitted: 28 July 2017

Accepted: 4 January 2018

Published: 12 March 2018

DOI : $10.19182 /$ remvt.31522

\section{Résumé}

Les perspectives de développement de l'élevage caprin semblent favorables en République démocratique du Congo. La connaissance des caractéristiques de l'élevage de la chèvre locale par les éleveurs est un facteur très important pour maîtriser sa production et la favoriser. L'objectif de cette étude a été d'améliorer la productivité de la chèvre locale de Mbanza-Ngungu en accroissant la connaissance de ses performances reproductives. Pour atteindre cet objectif, les données ont été collectées par observation directe des chèvres. Ces données ont permis de calculer par simulation les paramètres de reproduction et de dynamique de la population sur une période de cinq ans. L'étude a montré la pertinence du modèle pour simuler les caractères de reproduction de la chèvre de MbanzaNgungu et qu'il est inutile de garder les chèvres plus de cinq ans.

- Comment citer cet article : Gasigwa Sabimana R., Baenyi Simon P., Kizungu Vumilia R., 2017. Reproductive and population dynamics parameters of Mbanza-Ngungu's local goat in Democratic Republic of Congo. Rev. Elev. Med. Vet. Pays Trop., 70 (3): 93-97, doi: 10.19182/remvt.31522

\section{INTRODUCTION}

Les productions animales en zone tropicale, à l'instar de l'ensemble des productions agricoles, sont confrontées aux grands enjeux de la durabilité (Dedieu et al., 2011). Le développement de la population de chèvres allaitantes et laitières accompagne l'accroissement de la population humaine dans les zones tropicale et intertropicale. Du fait de leur grande adaptabilité, les caprins y sont élevés dans une gamme étendue de conditions agro-environnementales et selon un large éventail de systèmes de production, souvent traditionnels et peu artificialisés (Alexandre et al., 2012). Grâce à leur capacité d'adaptation élevée (Silanikove, 2000) et leur bon niveau de condition corporelle (Alexandre et

1. Université de Kinshasa, Faculté des sciences, Département math-info, Institut national de recherche agronomique (INERA), avenue des cliniques $n^{\circ} 13$, Kinshasa Gombé, République démocratique du Congo.

2. Université évangélique en Afrique, Bukavu, République démocratique du Congo.

3. Faculté des sciences agronomiques UNIKIN RDC, Direction de l'expérimentation agricole, INERA, Kinshasa Gombé, République démocratique du Congo.

* Auteur pour la correspondance

Tél. :+243814349930; +243899479219

E-mail : richardsabimana@gmail.com
Mandonnet, 2005 ; Browning et al., 2006), l'élevage des petits ruminants est considéré comme une source de revenu saisonnier pour les besoins de l'activité agricole et des ménages des fermiers en République démocratique du Congo. Cependant, les animaux sont souvent abandonnés à la divagation en milieu rural et leur productivité est très faible, $15 \%$ seulement sont exploités en élevage moderne de type intensif (Galvmed, 2014). L'avantage est que les perspectives de développement de l'élevage caprin semblent favorables dans le pays (Ahuya et al., 2009).

En milieu tropical les caractéristiques de l'élevage caprin connaissent d'énormes fluctuations saisonnières et interannuelles : une mortalité élevée et une réduction des performances laitières et reproductives surviennent en saison sèche. Une meilleure connaissance des performances de la chèvre locale permettrait de les améliorer. L'objectif de cette étude a ainsi été de d'évaluer les paramètres de reproduction et la dynamique d'une population de cette chèvre locale. Spécifiquement, il s'agissait :

- de présenter les différents paramètres de reproduction de la chèvre locale de Mbanza-Ngungu en RDC ;

- d'utiliser ces paramètres pour évaluer les performances de reproduction d'une chèvre de Mbanza-Ngungu laissée à la lutte pendant cinq ans avant de la réformer et évaluer l'évolution du nombre de têtes dans un cheptel lorsque l'élevage démarre avec une chèvre, deux chèvres... 100 chèvres mises à la lutte pendant cinq ans ; - de généraliser les résultats pour $n$ individus à la lutte. 


\section{MATERIEL ET METHODES}

\section{Milieu d'étude}

Létude a été menée à M'vuazi, dans la province du Kongo-Central, district des cataractes, territoire de la Mbaza-Ngungu, à environ 100 kilomètres au sud-ouest de Kinshasa. La température moyenne est de $24^{\circ} \mathrm{C}$, la pluviométrie moyenne annuelle est de 1472 millimètres et la saison sèche dure quatre à cinq mois (de mai à octobre).

\section{Echantillonnage}

Les données ont été collectées par observation d'un échantillon de 473 chèvres adultes ( 221 femelles et 252 mâles) sélectionnées par des chercheurs de l'INERA au cours du projet d'amélioration de la chèvre locale de 1986 à 1992. Nous avons utilisé des données secondaires issues du projet cité précédemment.

\section{Collecte des données}

Les paramètres suivants ont été collectés : l'identifiant, la souche, le lieu de mise bas, le rang de mise bas, le nombre de chevreaux par portée, l'âge de la mère, la date de la mise bas, la saison de la mise bas, le poids à la naissance, à 30 jours, 90 jours (au sevrage), 120 jours, 180 jours et à un an. Ces paramètres ont permis de calculer avec le logiciel $\mathrm{R}$ (version $\mathrm{R}$ i386 2.15.0) la date de la première saillie, la durée de gravidité, la proportion de gravidité gémellaire, l'intervalle entre mises bas, l'année de parturition, le nombre de mises bas, l'indice de fertilité, l'indice ou taux de fécondité, la productivité numérique, la période de réforme des bêtes, le nombre d'animaux dans le cheptel, le nombre de femelles et de mâles adultes, le nombre de chevreaux nés, le nombre d'immatures, le nombre de chevreaux sevrés, et la mortalité.

\section{Modélisation du système de reproduction}

Les expériences ont été menées dans un laboratoire informatique de l'INERA à Kinshasa. Il s'agissait d'évaluer les paramètres de reproduction de la chèvre locale de Mbanza-Ngungu (Mundondo), mise à la lutte pour une durée de cinq ans avant de la réformer, et de déduire la durée maximale de la période de reproduction garantissant une croissance positive du cheptel. Le modèle numérique a été décrit avec les hypothèses suivantes : une chèvre est mise à la lutte à l'âge moyen de 12 mois et la première mise bas intervient à l'âge moyen de 17 mois. Les algorithmes décrivant le modèle numérique émanant du système de reproduction de la chèvre sont détaillés ci-après :

\section{Algorithme de détermination du nombre de mise bas}

Posons i $\varepsilon$ \{mise bas simple, mise bas double, mise bas triple\}, nfl : le nombre de femelles mises à la lutte.

nb. de mises bas [i] $=(\mathrm{nfl} \times \mathrm{x}$ pourcentage de mises bas [i] $) / 100$

nb. de mises bas simples $=(\mathrm{nfl} \times$ pourcentage mises bas simples $) / 100$ nb. de mises bas doubles $=(\mathrm{nfl} \times$ pourcentage mises bas doubles $) / 100$ nb. de mises bas triples $=(\mathrm{nfl} \times$ pourcentage mises bas triples $) / 100$ nb. de mises bas $=n b$. de mises bas simples + nb. de mises bas doubles + nb. de mises bas triples

\section{Algorithme de détermination du nombre de petits nés} nb. de petits nés d'une mise bas simple $=$ nb. de mises bas simples nb. de petits nés d'une mise bas double $=$ nb. de mises bas doubles $\times 2$ nb. de petits nés d'une mise bas triple $=$ nb. de mises bas triples $x 3$ nb. total de petits nés $=$ nb. de petits nés d'une mise bas simple + nb. de petits nés d'une mise bas double + nb. de petits nés d'une mise bas triple.

Algorithme de détermination du nombre de femelles mises à la lutte au temps $i$

$\mathrm{nfl}[1]=\mathrm{nfl}$

$n f l[i+1]=n f l[i]+(n b$. total de petits nés $[i] / 2)$ où nfl désigne le nombre de femelles à la lutte et

nfl [i] le nombre de femelles mises à la lutte au temps $\mathrm{i}=\{1,2,3,4,5,6\}$.

Algorithme de détermination du nombre d'années par mise bas au temps $t$

AnParRate $=(365 / \mathrm{IMB})$

$\mathrm{NS}=$ AnParRate $\mathrm{x} 2$

FNAPB $=$ function $(\mathrm{t})(2 / \mathrm{NS}) \times \mathrm{t}$

$\mathrm{NAPB}=$ FNAPB $(\mathrm{t})$

où NS désigne une variable quelconque ; AnParRate (annual parturition rate) le nombre de parturitions par an ; IMB l'intervalle de parturition ; FNAPB désigne une fonction du nombre d'années par mise bas ; NAPB une fonction calculant le nombre d'années par mise bas au temps t.

Algorithme du calcul de l'âge de la mère à la mise bas au temps $t$

$\mathrm{ANt}=$ function $(\mathrm{t}) \mathrm{As} 1+\mathrm{dg}+(\mathrm{t}-1) \times \mathrm{IMB}$

$\mathrm{AMj}=\mathrm{ANt}(\mathrm{t})$

$\mathrm{AMm}=\mathrm{ANt}(\mathrm{t}) / 30$

où As1 = 360 jours désigne l'âge de la mise en saillie de la chèvre ; ANt une variable contenant une fonction calculant l'âge de la mère ; $\mathrm{dg}=150$ jours, la durée de gravidité ; AMj l'âge de la mère en jours ; AMm l'âge de la mère en mois.

\section{RESULTATS}

\section{Croissance et reproduction}

Dans la chèvrerie de M'vuazi à Mbaza-Ngungu, la chèvre atteint généralement son poids maximal à un an, âge de la première saillie (figure 1). Les jeunes boucs sont pubères vers cinq à six mois. La gravidité dure environ 145 à 150 jours et l'intervalle entre mises bas en moyenne 240 jours. En deux ans la chèvre a mis bas trois fois (tableau I).

La mise bas gémellaire dépend du rang de la portée (tableau II). Parmi les chèvres $36,2 \%$ ont mis bas deux petits lors de leur deuxième mise bas, et trois petits lors de leur sixième mise bas avec une probabilité de 6,8\% (tableau III). Selon les analyses effectuées, 18,2 \% des jumeaux ont été produits par des primipares et $81,8 \%$ par des pluripares. En regroupant les chèvres par le nombre de portées, 36,8 \% des pluripares ont mis bas des jumeaux, et $45 \%$ des triplés. Chez les primipares, $13 \%$ ont mis bas des jumeaux et $5,2 \%$ des triplés.

L'allaitement des chevreaux a duré entre 60 et 90 jours. Il est supposé qu'à l'âge de trois mois un chevreau peut se nourrir entièrement de fourrage de très haute qualité. De ce fait, séparer les chevreaux de leur mère à cet âge ne leur causera aucun préjudice et favorisera la reproduction dans le troupeau. Le poids moyen d'un chevreau a été de 1,6 kg à la naissance, il a atteint $7 \mathrm{~kg}$ au sevrage et 17,3 kg à la maturité (figure 2).

\section{Durée d'utilisation optimale par les reproducteurs}

Les chèvres étaient réformées à six ou sept ans, alors que les boucs l'étaient à quatre ou cinq ans. A 57 mois (soit 4 ans et 9 mois), la chèvre avait en général mis bas six fois. L'effectif total des chevreaux produits par celle-ci était environ de six petits (tableau I). La performance d'une chèvre mise à la lutte pendant cinq ans a été mesurée par le nombre de petits nés par mise bas. Le nombre total de petits nés par mise bas a diminué selon le rang de la mise bas ; il a été respectivement de 1,$4 ; 1,2 ; 1,0 ; 1,1 ; 0,9$ et 0,2 de la première à la sixième mise bas (figure 3). Cette diminution du nombre de petits nés par mise bas a continué jusqu'à déclarer la chèvre improductive, et à la classer dans le groupe des caprins à viande. Une chèvre était réformée après six à sept ans de service. En six ans, elle mettait bas en général 7,6 fois et avait 7,6 petits, et en sept ans elle mettait bas 8,8 fois et avait 8,8 petits. 
La productivité numérique était excellente à la première mise bas $(1,42)$ mais elle a chuté bien en dessous de $1(0,2$; tableau I) à la sixième mise bas. Par conséquent la chèvre locale de Mbanza-Ngungu peut être déclarée improductive après six mises bas (figure 3).

\section{Dynamique de la population sur une période de cinq ans}

En laissant une chèvre à la lutte pendant cinq ans, le nombre de femelles dans la chèvrerie a augmenté progressivement. De 1,7 femelle à partir de la deuxième mise bas, le nombre de futures femelles reproductives a atteint respectivement 2,$3 ; 2,8 ; 3,4$ et 3,9 (figure 4) de la troisième à la sixième année de lutte. Le taux de naissance des femelles a été de 45,5\%, celui des mâles de 54,5\%. Le taux de survie avant sevrage a été de 19,1\% chez les femelles et de $27,5 \%$ chez les mâles. Le taux de mortalité après sevrage a été de 7,6 \% chez les femelles et de 10,8 \% chez les mâles. Parmi les chevreaux nés $28 \%$ ont atteint l'âge pré-adulte, avec $11,5 \%$ de préadultes femelles et $16,7 \%$ de pré-adultes mâles. Le taux de mortalité à un an pour les mâles et les femelles a été de $5 \%$. Lindice de fertilité, de fécondité et la productivité numérique ont été respectivement de 95,$6 ; 1,4$ et 1,2 .

\section{Tableau I}

Performances des chèvres de Mbanza-Ngungu en RDC sur une période de cinq ans

\begin{tabular}{|c|c|c|c|c|c|c|c|c|c|c|c|c|}
\hline $\begin{array}{l}\text { Rang } \\
\text { de mise } \\
\text { bas }\end{array}$ & $\begin{array}{l}\text { Age de } \\
\text { la mère } \\
\text { (jours) }\end{array}$ & $\begin{array}{c}\text { Age de } \\
\text { la mère } \\
\text { (mois) }\end{array}$ & $\begin{array}{c}\text { Nb. } \\
\text { d'années } \\
\text { par mise } \\
\text { bas }\end{array}$ & $\begin{array}{c}\text { Nb. de } \\
\text { mises } \\
\text { bas } \\
\text { simples }\end{array}$ & $\begin{array}{c}\text { Nb. de } \\
\text { mises } \\
\text { bas } \\
\text { doubles }\end{array}$ & $\begin{array}{c}\text { Nb. de } \\
\text { mises } \\
\text { bas } \\
\text { triples }\end{array}$ & $\begin{array}{c}\mathrm{Nb} . \\
\text { total de } \\
\text { mises } \\
\text { bas }\end{array}$ & $\begin{array}{l}\text { Nb. de } \\
\text { petits } \\
\text { nés mise } \\
\text { bas } \\
\text { simple }\end{array}$ & $\begin{array}{c}\text { Nb. de } \\
\text { petits } \\
\text { nés mise } \\
\text { bas } \\
\text { double }\end{array}$ & $\begin{array}{l}\text { Nb. de } \\
\text { petits } \\
\text { nés mise } \\
\text { bas } \\
\text { triple }\end{array}$ & $\begin{array}{l}\mathrm{Nb} . \\
\text { total de } \\
\text { petits } \\
\text { nés }\end{array}$ & $\begin{array}{l}\text { Productivité } \\
\text { numérique } \\
\text { (PN) }\end{array}$ \\
\hline Un & 510 & 17 & 0,658 & 0,595 & 0,258 & 0,103 & 0,956 & 0,595 & 0,516 & 0,309 & 1,42 & 1,42 \\
\hline Deux & 750 & 25 & 1,315 & 0,258 & 0,362 & 0,07 & 0,69 & 0,258 & 0,724 & 0,21 & 1,192 & 1,192 \\
\hline Trois & 990 & 33 & 1,973 & 0,086 & 0,21 & 0,172 & 0,468 & 0,086 & 0,42 & 0,516 & 1,022 & 1,022 \\
\hline Quatre & 1230 & 41 & 2,630 & 0,03 & 0,094 & 0,31 & 0,434 & 0,03 & 0,188 & 0,93 & 1,148 & 1,148 \\
\hline Cinq & 1470 & 49 & 3,288 & 0,018 & 0,053 & 0,275 & 0,346 & 0,018 & 0,106 & 0,825 & 0,949 & 0,949 \\
\hline Six & 1710 & 57 & 3,945 & 0,011 & 0,012 & 0,068 & 0,091 & 0,011 & 0,024 & 0,204 & 0,239 & 0,239 \\
\hline Total & & & & 0,998 & 0,989 & 0,998 & 2,985 & 0,998 & 1,978 & 2,994 & 5,97 & \\
\hline
\end{tabular}

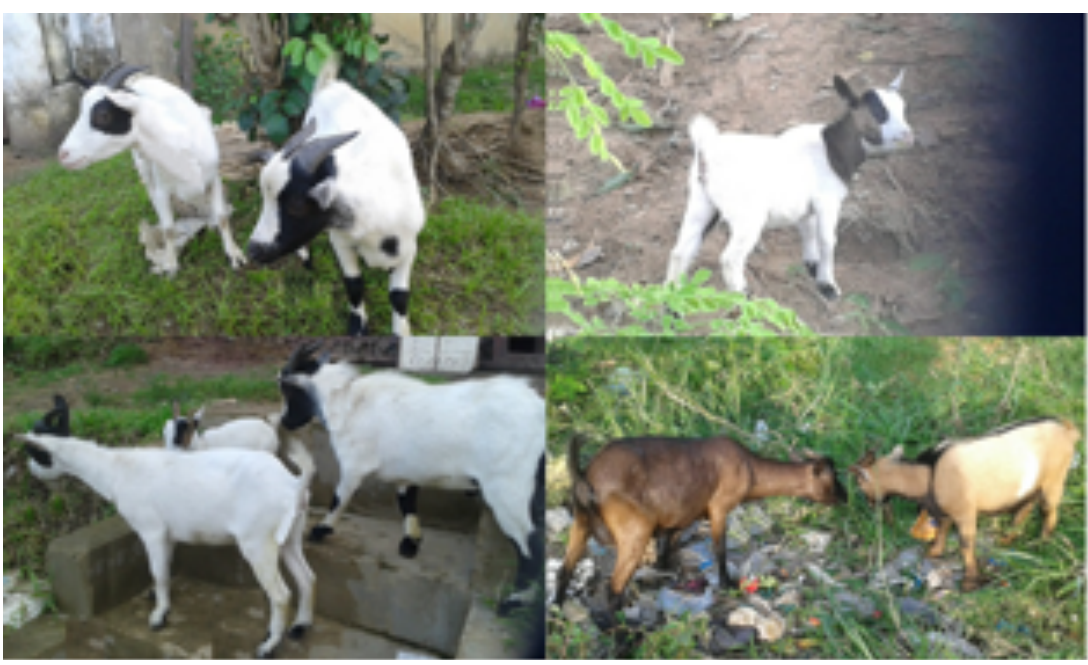

Tableau II

Taille de la portée des chèvres de Mbanza-Ngungu en RDC

\begin{tabular}{|cccc}
$\begin{array}{c}\text { Mise bas } \\
\text { Simple } \\
(\%)\end{array}$ & $\begin{array}{c}\text { Mise bas } \\
\text { double } \\
(\%)\end{array}$ & $\begin{array}{c}\text { Mise bas } \\
\text { triple (\%) }\end{array}$ & $\begin{array}{c}\text { Mise bas } \\
\text { quadruple } \\
(\%)\end{array}$ \\
\hline 37,02 & 49,62 & 12,60 & 0,76 \\
\hline
\end{tabular}

Figure 1 : chèvres de Mbanza-Ngungu, République démocratique du Congo.

Tableau III

Mises bas chez les chèvres de Mbanza-Ngungu à en RDC

\begin{tabular}{lccccccccccccccccccc} 
& \multicolumn{4}{c}{ Mise bas simple } & \multicolumn{1}{c}{ Mise bas double } & \multicolumn{1}{c}{ Mise bas triple } \\
\hline Rang de mise bas & 1 & 2 & 3 & 4 & 5 & 6 & 1 & 2 & 3 & 4 & 5 & 6 & 1 & 2 & 3 & 4 & 5 & 6 \\
$\%$ & 59,5 & 25,8 & 8,6 & 3 & 1,8 & 1,1 & 25,8 & 36,2 & 21 & 9,4 & 5,3 & 1,2 & 10,3 & 7 & 17,2 & 31 & 27,5 & 6,8
\end{tabular}


Si le fermier commence une chèvrerie avec 100 chèvres à la lutte, à la deuxième lutte il aura 171 femelles, ensuite respectivement 230, 281, 339, 386 femelles, de la troisième à la sixième lutte. Par conséquent l'indice de fertilité, l'indice de fécondité et la productivité numérique ne changent pas après cinq ans quel que soit le nombre de chèvres à mettre à la lutte pour cette race locale.

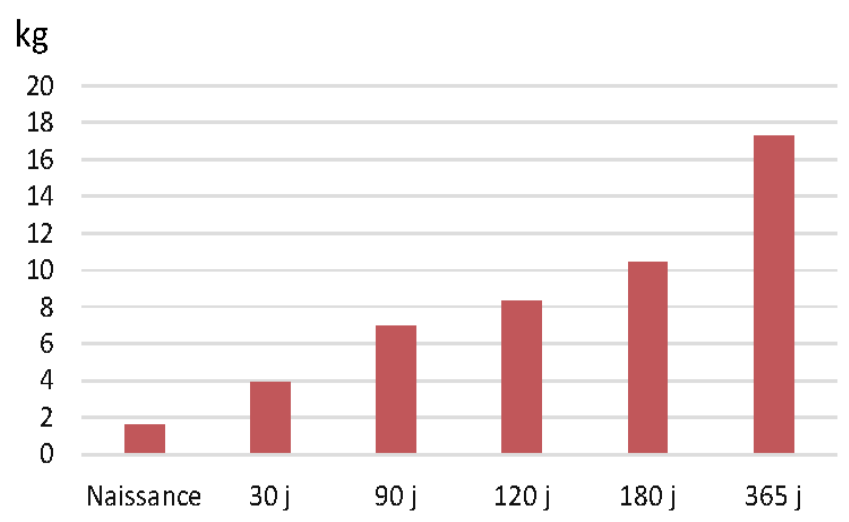

Figure 2 : poids moyen en fonction de l'âge des chèvres de Mbanza-Ngungu, République démocratique du Congo.

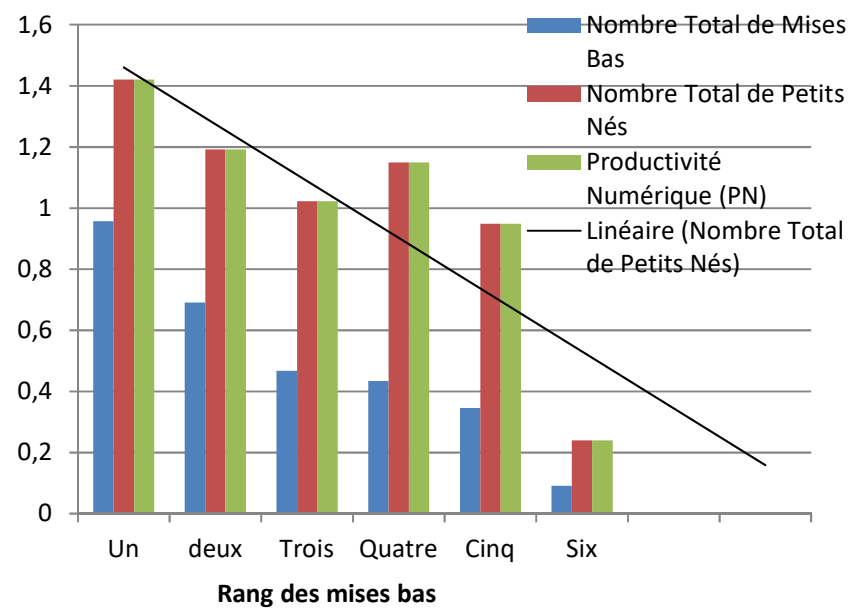

Figure 3 : évaluation de la performance en fonction du rang de mise bas des chèvres de Mbanza-Ngungu, République démocratique du Congo.

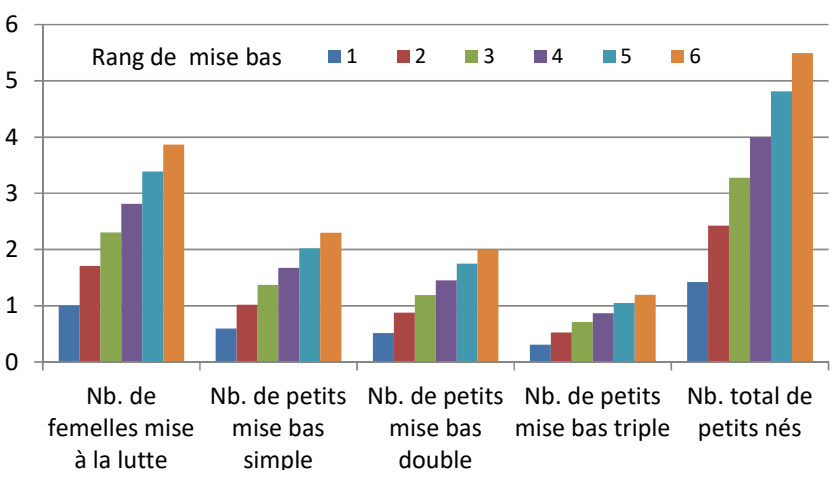

Figure 4 : dynamique de la population de chèvres de MbazaNgungu en RDC suivant le rang de mise bas répartie selon la portée.

\section{DISCUSSION}

En dépit de quelques divergences, les valeurs des paramètres de la reproduction de la chèvre simulées par le modèle ont été dans l'ensemble en accord avec les valeurs mesurées. La simulation par modèle a abouti à un accord plausible avec les données expérimentales, malgré la complexité du scénario de simulation et les propriétés de la reproduction de la chèvre.

Ces résultats sont similaires à ceux de Jansen et Van den Burg (2004) qui montrent que la puberté d'une chèvre est fortement liée à son poids, au mois de naissance et à sa race. Ils sont par ailleurs similaires à ceux de Malher et al. (1999) qui montrent que la réforme d'une fraction du troupeau a un coût qui doit être réduit pour pouvoir assurer la rentabilité de l'exploitation : pour améliorer la production, il est préférable de laisser une chèvre fertile à la lutte dans un troupeau pendant cinq ans au maximum. La première mise en saillie est effectuée à l'âge d'un an. Pour avoir un bon taux de fécondation, les femelles sont sélectionnées et l'alimentation est bien surveillée (Browning et al., 2006), avec une proportion mâle/femelle de 1 pour 30 (Huart, 1987). Une bonne alimentation augmente le taux de gravidité gémellaire (Huart, 1987). Plus la chèvre est grande et en bonne santé, plus grande est la probabilité qu'elle fasse une gravidité gémellaire. Cela s'explique par le fait que la croissance d'une jeune chèvre mature se poursuit en parallèle avec les mises bas et le poids de la mère gravide entre en jeu pour assurer le partage des ressources avec ses petits. A M'vuazi l'intervalle entre mises bas moyen a été de 240 jours. Il est bon selon Huart (1987), il serait mauvais à 180 jours et excellent à 360 jours. Après une période d'un an, la chèvre met bas environ 1,5 fois par an, c'est à dire trois mises bas en deux ans.

La durée de lactation pour la chèvre de Mbanza-Ngungu a été comprise entre 60 et 90 jours. D'autres auteurs, comme Williams (1990), Ahuya et al. (2009), Browning et al. (2006), mentionnent 60 à 120 jours. Ce trait est important pour une production améliorée, il est plus long pour les chèvres laitières (Gaddour et al., 2008) mais la chèvre locale de Mbanza-Ngungu n'est pas une chèvre laitière, c'est une chèvre à viande.

La mortalité a été très élevée dans le troupeau ; une amélioration de l'élevage est nécessaire. Plusieurs facteurs étaient à l'origine de la mortalité des chevreaux avant sevrage, notamment les maladies (Richard, 2007), l'alimentation, le logement, le climat (Chemineau, 1985). Selon Williams (1990), une mortalité avant sevrage de 15 à $25 \%$ est acceptable, elle est excessive si le taux atteint $40 \%$. Selon cet auteur, le taux de passage de l'étape sevrage est excellent à $85 \%$, il est bon à $75 \%$ et mauvais à $60 \%$. Beaucoup de chevreaux sont morts très jeunes parce que les chèvres à M'vuazi vivent en liberté et ainsi le rendement a été faible au moment du sevrage. Ces résultats sont similaires à ceux de Chemineau (1985) : dans une chèvrerie évoluant dans les conditions de vagabondage, on note en particulier un taux de mortalité avant sevrage très élevé (plus de $50 \%$ ) ayant en général pour cause divers accidents comme la noyade, l'empoisonnement ou se faire écraser par un véhicule.

\section{CONCLUSION}

Les résultats, en particulier la mise en évidence d'une forte mortalité, devraient ouvrir la voie à des recherches visant l'amélioration de la production de la chèvre locale en République démocratique du Congo. Pour améliorer les résultats de cet élevage où la mortalité est très élevée, il faut réformer le système d'élevage des caprins en RDC et encourager la recherche sur les chèvres élevées dans cette zone. Les résultats obtenus montrent la pertinence du modèle pour simuler les performances de la reproduction d'une chèvre laissée à la lutte pendant cinq ans. Les algorithmes utilisés pour évaluer le nombre de bêtes dans la chèvrerie sont inefficaces au-delà de cinq ans mais leur utilisation montre qu'il faut éviter de garder inutilement une chèvre improductive dans un cheptel de production. 


\section{REFERENCES}

Ahuya C.O., Ojango J.M.K., Mosi R.O., Peacock C.P., Okeyo A.M., 2009. Performance of Toggenburg dairy goats in smallholder production systems of the eastern highlands of Kenya. Small Rum. Res., 83: 7-13, doi: 10.1016/j.smallrumres.2008.11.012

Alexandre G., Arquet R., Fleury J., Boval M., Archimède H., Mahieu M., Mandonnet N., et al., 2012. Systèmes d'élevage caprins en zone tropicale : analyse des fonctions et des performances. Prod. Anim., 25 (3) : 305-316

Alexandre G., Mandonnet N., 2005. Goat meat production in harsh environments. Small Rumin. Res., 60: 53-66, doi: 10.1016/j.smallrumres.2005.06.005

Browning R., Payton T., Donnelly B., Leite-Browning M.L., Pandya P., Hendrixson W., Byars M., 2006. Evaluation of three meat goat breeds for doe fitness and reproductive performance in the Southeastern United States. In: 8th World Congr. Genetics Applied to Livestock Production, 13-18 Aug. 2006, Belo Horizonte (Minas Gerais), Brazil

Chemineau P., Grude A., Varo H., 1985. Mortalité, poids à la naissance et croissance de chevreaux créoles nés en élevage semi-intensif. Ann. Zootech., 35 (2) : 193-204, doi : 10.1051/animres:19850205

Dedieu B., Aubin J., Duteurtre G., Alexandre G., Vayssières J., Bommel P., Faye B., et al., 2011. Conception et évaluation de systèmes d'élevage durables en régions chaudes à l'échelle de l'exploitation. Prod. Anim., 24 (nº spécial) : 113-128
Gaddour A., Najari S., Ouni M., 2008. Improving goat dairy production by grading up in an oasis of South Tunisia. Rev. Elev. Med. Vet. Pays Trop., 61 (1) : 57-62, doi : 10.19182/remvt.10014

Galvmed, 2014. La chèvre, l'élevage de choix pour le paysan en RDC. www.ecocongo.cd (consulté 26 déc. 2014)

Huart A., 1987. La chèvre au Zaïre, guide pour l'éleveur. Université de Lubumbashi, Lubumbashi, République démocratique du Congo, 75 p.

Jansen C., Van den Burg K., 2004. L'élevage de chèvres dans les zones tropicales. Digigrafi, Wageningen, Pays-Bas

Malher X., Beaudeau F., Poupin B., Falaise G., Losdat J., 1999. Réforme et renouvellement dans les grands troupeaux laitiers caprins de l'ouest de la France. Prod. Anim., 12 (2) : 123-133

Richard C., 2007. Les maladies du chevreau. www.alliance-elevage.com/ f134_Maladies_du_chevreau_lire_la_suite.pdf

Silanikove N., 2000. The physiological basis of adaptation in goats to harsh environments. Small Rum. Res., 35: 181-193, doi: 10.1016/j. smallrumres.2014.11.005

Williams T.O., 1990. Scope for improvement of small ruminant production in the Sahel. In: Social science research for agricultural technology development spatial and temporal dimensions (Ed. Dvorak K.A.). International Institute of Tropical Agriculture, Ibadan, Nigeria

\section{Summary}

Gasigwa Sabimana R., Baenyi Simon P., Kizungu Vumilia R. Reproductive and population dynamics parameters of MbanzaNgungu's local goat in Democratic Republic of Congo

Prospects for the development of goat breeding in the Democratic Republic of Congo seem favorable. Knowledge of the characteristics of local goat farming by breeders is a very important factor to control and promote goat production. The objective of this study was to improve the productivity of Mbanza-Ngungu's local goat by increasing the knowledge of its reproductive performance. To achieve this objective, data were collected by direct observation of the goats. These data were used to simulate reproductive and population dynamics parameters over a five-year period. The study showed the relevance of the model to simulate the reproduction traits of Mbanza-Ngungu's goats and that it is useless to keep them beyond five years.

Keywords: goat, small ruminant, reproduction, population dynamics, Democratic Republic of Congo

\section{Resumen}

Gasigwa Sabimana R., Baenyi Simon P., Kizungu Vumilia R. Parámetros reproductivos y de dinámicas de población de la raza local de cabra de Mbanza-Ngungu en la República Democrática del Congo

Los prospectos para el desarrollo de la cría de cabras en la República Democrática del Congo parecen favorables. El conocimiento de las características de la cría local de cabras que utilizan los criadores es un factor importante para controlar y promover la producción. El objetivo del presente estudio fue de mejorar la productividad de la cabra local de Mbanza-Ngungu, mediante un aumento del conocimiento de sus rendimientos reproductivos. Para lograr este objetivo, se colectaron datos mediante observación directa de las cabras. Estos datos fueron utilizados para simular los parámetros reproductivos y de dinámica de la población durante un periodo de cinco años. Este estudio mostró la aptitud del modelo para simular los trazos de reproducción de la cabra de Mbanza-Ngungu y que es inservible mantener cabras por más de cinco años.

Palabras clave: caprino, pequeño rumiante, reproducción, dinámica de problaciones, República democrática del Congo 
